\title{
Standard Prophylactic Strategy against Peritoneal Dissemination Metastasis in Gastric Cancer
}

\author{
Satoshi Ikeshima ${ }^{1}$, Masafumi Kuramoto ${ }^{1}$, Shinya Shimada ${ }^{1}$, Kenichiro Yamamoto $^{1}$, \\ Toshiro Masuda ${ }^{1}$, Tatsunori Miyata ${ }^{1}$, Shinichi Yoshimatsu' ${ }^{2}$, Masayuki Urata ${ }^{2}$, Hideo Baba ${ }^{3}$ \\ ${ }^{1}$ Department of Surgery, Yatsushiro Social Insurance General Hospital, Yatsushiro, Japan; ${ }^{2}$ Department of Gastroenterology and \\ Hepatology, Yatsushiro Social Insurance General Hospital, Yatsushiro, Japan; ${ }^{3}$ Department of Gastroenterological Surgery, Graduate \\ School of Medical Sciences, Kumamoto University, Kumamoto, Japan. \\ Email: Ikeshima@yatsushiro-gh.jp
}

Received November $27^{\text {th }}, 2012$; revised December $29^{\text {th }}, 2012$; accepted January $8^{\text {th }}, 2013$

\begin{abstract}
Peritoneal carcinomatosis is the most frequent pattern of metastasis and recurrence in patients with gastric cancer, and the prognosis of those patients with peritoneal metastasis is extremely poor. Once peritoneal metastasis is formed, it is extremely difficult to overcome. EIPL (extensive intraoperative peritoneal lavage) is a quite useful and practical adjuvant surgical technique for the gastric cancer patients who are likely to suffer from peritoneal recurrence. EIPL includes 10 times of an extensive shake and wash of abdominal cavity with saline followed by the complete aspiration of the fluid after potentially curative operation, which is supposed to have an amazing cyto-reduction power. The purpose of this article is to review the effect of EIPL on prevention of peritoneal recurrence in the patients with peritoneal free cancer cells and to evaluate its validity as a standard prophylactic strategy against peritoneal recurrence in gastric cancer.
\end{abstract}

Keywords: Gastric Cancer; Intra-Peritoneal Free Cancer Cells; Peritoneal Metastasis; EIPL

\section{Introduction}

Recent advances in diagnostic and surgical techniques have improved the prognosis of early gastric cancer, whereas advanced gastric cancer still remains a lifethreatening disease. Peritoneal carcinomatosis is the most frequent pattern of metastasis and recurrence in patients with gastric cancer, and the prognosis of the patients with peritoneal metastasis is extremely poor [1-5]. The median survival time (MST) of such patients is reported to be 3 - 6 months [6], and a standard regimen against peritoneal metastasis of gastric cancer has not yet been established [7-10].

Stage IV gastric cancer, including peritoneal metastasis, is considered incurable and this population is usually ineligible for radical operation. Recommended treatments for this population are commonly thought to be chemotherapy, palliative surgery and palliative care [11]. Patients with cytology-positive peritoneal lavage fluid and without macroscopic peritoneal metastasis of gastric cancer $(\mathrm{CY}+/ \mathrm{P}-)$ are classified as Stage IV like patients with overt peritoneal metastasis. It has been reported that the outcome of the $\mathrm{CY}+/ \mathrm{P}-$ patients is extremely poor and almost the same as that of patients with peritoneal metastasis, however, whether those patients should be treated radically or palliatively is still a controversial issue [12-14].

It is already generally accepted that peritoneal metastasis is completed by implantation of peritoneal free cancer cells exfoliated from serosa-invasive tumors through the process of fixation and progression of cancer cells on the peritoneum. The situation of $\mathrm{CY}+/ \mathrm{P}-$ could possibly mean a condition where the implantation of cancer cells to the peritoneal wall has not yet occurred, and therefore, it is thought that there are apparent differences between the condition of $\mathrm{CY}+/ \mathrm{P}-$ and peritoneal metastasis. From this point of view, the situation of $\mathrm{CY}+\mathrm{P}-$ might be the last opportunity for surgeons to undertake some countermeasures for the peritoneal metastasis to rescue those miserable patients.

In this article, we reviewed the efficacy and advantage of our new adjuvant intraoperative method for reduction of the peritoneal recurrence, and clarified the feasibility and validity of adopting this method as a standard prophylactic strategy for the prevention of peritoneal metastasis in advanced gastric cancer.

\section{Theory of EIPL (Extensive Intraoperative Peritoneal Lavage)}

Peritoneal metastasis is mainly caused by seeding of the free cancer cells originated from the primary gastric can- 
cer, especially that of serosa-invasive tumors. The free cancer cells need some time to get over the steps of attaching and proliferating on the peritoneal wall until peritoneal metastasis is completed. So, there would still remain something that we can do. If this supposition is true, the number of free cancer cells could be reduced to potentially zero by just like the so-called "limiting dilution method". Applying this theory, we have proposed "extensive intraoperative peritoneal lavage" (EIPL) for reducing the number of intraperitoneal free cancer cells as a useful intraoperative technique [15].

Briefly, the peritoneal cavity is extensively stirred and washed after the potentially curative operation, which is followed by the complete aspiration of the fluid. This procedure is done 10 times using $1 \mathrm{~L}$ of physiological saline. 10 washes of a 1:10 dilution result in just 1 cancerous cell from $10^{10}$ cells in the abdominal cavity. Furthermore, sufficient stirring and washing of the abdominal cavity would remove the cancer cells which merely adhere to the peritoneum.

As a pilot study, we performed EIPL to the five cases of serosa-invasive gastric cancer with $\mathrm{CY}+/ \mathrm{P}-$, and its efficacy was evaluated by the ultra-rapid quantitative RT-PCR protocol. Sequential washing of intraperitoneal free cancer cells of $3.8 \times 10^{5} \pm 1.4 \times 10^{5} / 100 \mathrm{ml}$ of lavage decreased the number to $2.8 \pm 1.5$ cells by 6 to 8 washes.

Free cancer cells were not detected in the fluid after that (Figure 1). On the other hand, $2.8 \times 10^{4} \pm 4.5 \times 10^{4}$ of intraperitoneal free cancer cells still remained in 100 $\mathrm{ml}$ of the lavage when not treated with EIPL [16].

Our preliminary subset analysis based on 22 consecutive patients with $\mathrm{CY}+/ \mathrm{P}-$ who underwent curative surgi-

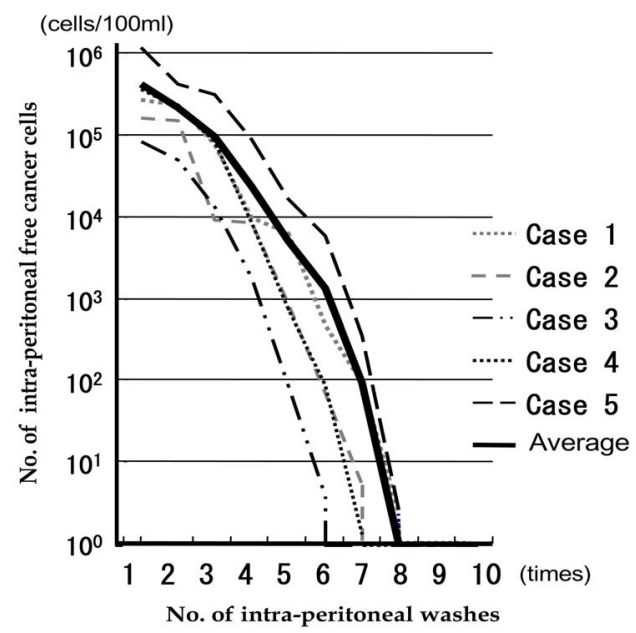

Figure 1. Changes in numbers of intraperitoneal free cancer cells in five gastric cancer patients with $\mathrm{CY}+$ treated by EIPL therapy. The numbers of free cancer cells in $100 \mathrm{ml}$ of samples from the lavage fluid using 1 liter of saline were measured by ulra-rapid RT-PCR. The free cancer cells were serially diluted by 8 liters of saline saline and disappeared in washing fluid after the 8 th wash. cal treatment for advanced gastric cancer, and who were followed up for 2 years or until death, has shown a statistically significant improvement of a 2-year survival rate when treated with EIPL as compared with when not treated with EIPL [15].

\section{Application of EIPL to a Clinical Study}

Based on our pioneering study, we have advocated EIPL-IPC (intraperitoneal chemotherapy) therapy. After the EIPL treatment, cisplatin (CDDP) is administrated into the abdominal cavity at a dose of $100 \mathrm{mg} /$ body and the solution is drained 1 hour after the injection. In this way, even if only a few cancer cells were to remain, these cells might find it difficult to survive and/or to disseminate due to the effects of IPC.

To clarify the distinct survival effects of EIPL-IPC therapy, we designed a prospective randomized multicenter trial for advanced gastric cancer patients with $\mathrm{CY}+/ \mathrm{P}-$ [17]. A total of 88 gastric cancer patients with $\mathrm{CY}+/ \mathrm{P}-$ from 1522 patients with advanced gastric cancer at multicenters were enrolled in this study, and were randomly allocated to three groups: surgery alone group, surgery plus intraperitoneal chemotherapy (IPC) group, and surgery plus EIPL and IPC (EIPL-IPC) group. Peritoneal lavage for the surgery alone group and the IPC group was done with 3 liters of saline (1 liter, three times) before the closure of the abdominal wall or IPC, respectively.

The overall 5-year survival rate of patients with EIPLIPC was $43.8 \%$, and this data was significantly higher than that of the IPC group $(4.6 \%, \mathrm{P}<0.0001)$ and the surgery alone group $(0 \%, \mathrm{P}<0.0001)$, as shown in Figure 2. Among various recurrent patterns, the EIPL-IPC group had a significantly lower incidence of peritoneal recurrence than either of the other groups. Univariate analysis identified lymph node metastasis, lymphovascular invasion, and EIPL as significant prognosis factors. Further multivariate analyses with these 3 factors as vari-

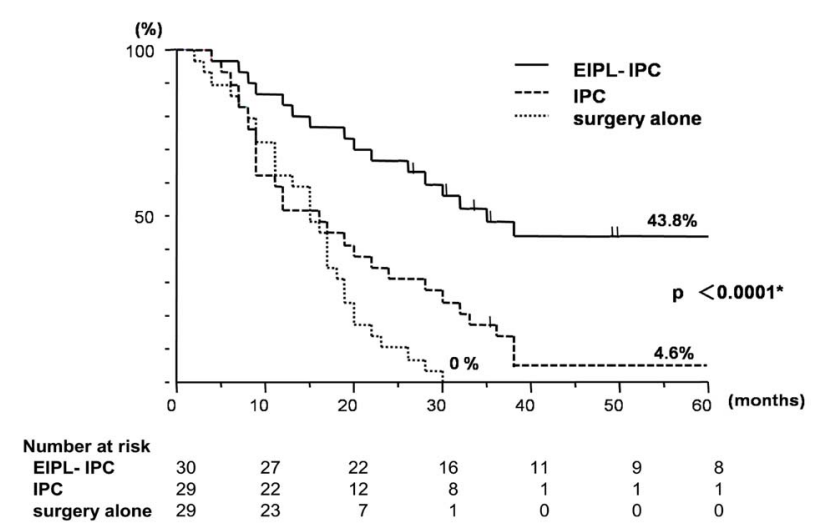

Figure 2. The survival curves for the 88 patients stratified according to the treatments. ${ }^{*}$ By log-rank test. 
ables clearly revealed that EIPL was the most significant impact factor (Table 1).

The results of this study far exceeded our expectations and showed a remarkably better prognosis than previous studies on gastric cancer patients with $\mathrm{CY}+/ \mathrm{P}-$. For example, a study on the median survival time (MST) of 91 patients with $\mathrm{CY}+\mathrm{P}-$ who had potentially curative operations stated survival to be only 386 days [18], and the 5 -year overall survival rate has been $13 \%$ [19]. In our study, the surgery alone group as well as the IPC group also showed similar results to the reports just cited. Surprisingly, however, in the EIPL-IPC group the overall 5-year survival rate and MST were $42.1 \%$ and 35 months, respectively, remarkably significant improvement of both survival and MST. These results were convincing and are promising enough to serve as a solid basis on which to build strong confidence in and high expectations for employing the EIPL-IPC therapy.

\section{Expanding Adaptation of EIPL}

We could find remarkable effectiveness of EIPL with improving the survival of the $\mathrm{CY}+/ \mathrm{P}-$ gastric cancer after curative surgery. The power of its cyto-reduction in the abdominal cavity is amazing and splendid, so further application of EIPL was considered.

Despite neither the apparent existence of abdominal free cancer cells nor overt peritoneal metastasis, approximately half of patients with serosa-involved gastric cancer developed peritoneal recurrence after curative operations [20]. In addition, some non-serosa-involved gastric cancers advance to peritoneal recurrence, even

Table 1. Survival analysis of variables predicting predicting risk of death for patients with $\mathrm{CY}+(\mathbf{n}=\mathbf{8 8})$.

\begin{tabular}{lll}
\hline Variable & $\begin{array}{c}\text { Univariate } \\
\text { P value }\end{array}$ & $\begin{array}{c}\text { Multivariate } \\
\text { P value }\end{array}$ \\
\hline Sex & 0.156 & \\
Male/Female & 0.976 & \\
Age & & \\
Type of gastrectomy & & \\
Total/Distal & 0.658 & \\
Histologic type & & \\
tub2/por2/sig & & \\
Depth of tumor invasion & 0.363 & \\
ss/se/si & & \\
Lymph node metastasis & $0.0009^{*}$ \\
N0/N1/N2/N3 & & \\
Borrmann's type & 0.408 & \\
II/III/IV & & 0.0071 \\
Lymphovascular invasion \\
ly1/ly2/ly3 \\
v1/v2/v3
\end{tabular}

*Statistically significance; EIPL: extensive intraoperative peritoneal lavage; IPC: intraperitoneal chemotherapy. though a curative operation has been performed [5,2123]. We elucidated the mechanisms of peritoneal recurrence after curative operations for patients with non-serosa-involved gastric cancer.

CEA and CK20 mRNA in the peritoneal lavage samples from 63 patients with non-serosa-involved gastric cancer which were obtained just after laparotomy and after lymph node dissection were examined by an ultrarapid quantitative RT-PCR system [16]. In the peritoneal lavage samples from non-serosa-involved cases after lymph node dissection, CEA or CA20 mRNA were detected in 16 of 63 patients $(25.4 \%)$ despite no detection of either CEA or CA20 mRNA just after laparotomy. These were not evident in the mucosal (M) tumor, but were detected in three $(14.3 \%)$, six $(46.2 \%)$ and seven $(53.8 \%)$ patients with submucosal (SM), muscularis propria (MP) and subserosal (SS) tumors, respectively. These data suggested the existence of free cancer cells in the peritoneal cavity after lymph node dissection with non-serosa-involved gastric cancer patients. Moreover, our previous study on 1272 gastric cancer patients revealed that $1 / 257$ cases $(0.4 \%)$ of SM and $6 / 136$ cases (4.4\%) of MP developed peritoneal recurrences after potentially curative resections [23]. Among them, $86 \%$ of the patients had lymph node metastasis and/or lymphatic invasion. Our results demonstrated that lymph node dissection would be a main factor for spreading viable free cancer cells into the peritoneal cavity. Thus, we came to an assurance that lymph node dissection itself is a cause of peritoneal dissemination, seeding viable cancer cells from the lymphatic vessels to the abdominal cavity. As there should be a low risk of the completion of peritoneal metastasis in such cases with non-serosal-involved gastric cancer, EIPL therapy will demonstrate its effectiveness to the maximum on the prevention of peritoneal recurrences after curative operations.

\section{EIPL as a Standard Prophylactic Technique for Prevention of Peritoneal Recurrence in Gastric Cancer}

Peritoneal metastasis, which often arises in patients with advanced gastric cancer, is well known as a miserable and ill-fated disease even though curative surgery is performed. Once peritoneal metastasis is formed, it is extremely difficult to defeat. Many investigators have made strenuous efforts to overcome this horrible disease; however, we have to say that there are not any satisfactory reports concerning effective measures for peritoneal metastasis. That's why we have advocated that some effective measures are needed before peritoneal metastasis is completed.

The situation of $\mathrm{CY}+/ \mathrm{P}-$ means the condition where the implantation of free cancer cells derived from the primary tumor has not yet occurred. We suppose there 
should be apparent differences between the conditions of $\mathrm{CY}+/ \mathrm{P}-$ and peritoneal metastasis which would require different management strategy. Therefore, it is considered reasonable and relevant to focus on devising some effective surgical measures to prevent peritoneal recurrence, accompanied by appropriate and respectable radical resection. Although the Dutch report has described the high post-operative morbidity and mortality after gastrectomy with D2 lymph node dissection [24], radical resections with D2 lymphadenectomy appear to be feasible and safe for patients in Japan [25-28]. In our study, operative morbidity and mortality was $1.5 \%$ and $0.5 \%$, respectively. These results show that potential benefits of D2 operations would outweigh the risk of morbidity and mortality after the radical operation. Complete extirpation of gastric cancer with a sufficient resection margin from the tumor and removal of metastatic lymph nodes is the only measure that could bring the hope of cure for patients with gastric cancer [1,29-32], therefore, advanced gastric cancer should be treated with radical resection even if it is accompanied by $\mathrm{CY}+/ \mathrm{P}-$ because our novel EIPL-IPC regimen would have the power to cancel the $\mathrm{CY}+$ condition.

The innovative EIPL is very practical and its theoretical basis creates high expectations as to the effects of cyto-reduction, potentially to zero. Furthermore, EIPL is simple, not time-consuming, inexpensive, and it is not curtailed by place or time, so can easily be performed anytime, anywhere. Also, it does not require the use of any special techniques or devices. In addition, a point worthy of special mention is that EIPL itself is completely harmless to patients.

\section{Conclusion Equation}

We reviewed clinical studies concerning EIPL, demonstrating its amazing ability to improve the survival of gastric cancer patients with peritoneal free cancer cells. These results convinced us to advocate EIPL as an optimal treatment for gastric cancer patients who are likely to suffer from peritoneal metastasis; a miserable and illfated disease. It is our earnest wish that many surgeons would adopt EIPL worldwide as a prophylactic strategy for peritoneal metastasis.

\section{REFERENCES}

[1] D. C. Balfour, "Factor of Significance in the Prognosis of Cancer of the Stomach," Annals of Surgery, Vol. 105, No. 5, 1937, pp. 733-740. doi:10.1097/00000658-193705000-00009

[2] Y. Kodera, H. Nakanishi, Y. Yamamura, et al., "Prognostic Value and Clinical Implications of Disseminated Cancer Cells in the Peritoneal Cavity Detected by Reverse Transcriptase-Polymerase Chain Reaction and Cytology,"
International Journal of Cancer, Vol. 79, No. 4, 1998, pp. 429-433. doi:10.1002/(SICI)1097-0215(19980821)79:4<429::AIDIJC20>3.0.CO;2-Z

[3] M. Ikeguchi, S. Matsumoto, S. Yoshida, et al., "Laparoscopic-Assisted Intraperitoneal Chemotherapy for the Patients with Scirrhous Gastric Cancer," Chemotherapy, Vol. 51, No. 1, 2005, pp. 15-20. doi: 10.1159/000084018

[4] M. Hiratsuka, T. Iwanaga, H. Furukawa, et al., "Important Prognostic Factors in Surgically Treated Gastric Cancer Patients," Cancer \& Chemotherapy, Vol. 22, No. 5, 1995, pp. 703-708.

[5] C. H. Yoo, S. H. Noh, D. W. Shin, et al., "Recurrence Following Curative Resection for Gastric Carcinoma," The British Journal of Surgery, Vol. 87, No. 2, 2000, pp. 236-242. doi:10.1046/j.1365-2168.2000.01360.x

[6] Y. Kitamura, K. Hayashi, T. Sasagawa, et al., "Pilot Study of S-1 in Patients with Disseminated Gastric Cancer," Drugs under Experimental and Clinical Research, Vol. 29, No. 3, 2003, pp. 125-130.

[7] C. Kunisaki, H. Shimada, H. Akiyama, et al., "Therapeutic Outcomes of Continuous Hyperthermic Peritoneal Perfusion against Advanced Gastric Cancer with Peritoneal Carcinomatosis," Hepato-Gastroenterology, Vol. 53, No. 69, 2006, pp. 473-478.

[8] C. Kunisaki, H. Shimada, M. Nomura, et al., "Lack of Efficacy of Prophylactic Peritoneal Perfusion on Subsequent Peritoneal Recurrence and Survival in Patients with Advanced Gastric Cancer," Surgery, Vol. 131, No. 5, 2002, pp. 521-528. doi:10.1067/msy.2002.123769

[9] S. Ishizoe, F. Maruta, H. Saito, et al., "Efficacy of S-1 for Patients with Peritoneal Metastasis of Gastric Cancer," Chemotherapy, Vol. 52, No. 6, 2006, pp. 301-307. doi:10.1159/000096002

[10] L. Suhsien, E. M. Bart, G. M. Stuart, et al., "Results Following Resection for Stage IV Gastric Cancer; Are Better Outcomes Observed in Selected Patients Subgroups?" Journal of Surgical Oncology, Vol. 95, No. 2, 2007, pp. 118-122. doi: $10.1002 /$ jso.20328

[11] Japanese Gastric Cancer Association, "Gastric Cancer Treatment Guidelines," 3rd Edition, Kanehara, Tokyo, 2010.

[12] T. Boku, Y. Nakane, T. Minoura, et al., "Prognostic Significant of Serosal Invasion and Free Cancer Cells in Gastric Cancer," The British Journal of Surgery, Vol. 77, No. 4, 1990, pp. 436-439. doi:10.1002/bjs. 1800770425

[13] E. Bando, Y. Yonemura, Y. Takeshita, et al., "Intraoperative Lavage for Cytological Examination in 1297 Patients with Gastric Carcinoma," American Journal of Surgery, Vol. 178, No. 3, 1999, pp. 256-262. doi:10.1016/S0002-9610(99)00162-2

[14] J. J. Mezhir, M. A. Shah, L. M. Jack, et al., "Positive Peritoneal Cytology in Patients with Gastric Cancer; Natural History and Outcome of 291 Patients," Annals of Surgical Oncology, Vol. 17, No. 12, 2010, pp. 3173-3180. doi:10.1245/s10434-010-1183-0

[15] S. Shimada, E. Tanaka, T. Marutsuka, et al., "Extensive Intraoperative Peritoneal Lavage and Chemotherapy for 
Gastric Cancer Patients with Peritoneal Free Cancer Cells," Gastric Cancer, Vol. 5, No. 3, 2002, pp. 168-172. doi:10.1007/s101200200029

[16] T. Marutsuka, S. Shimada, K. Shiomori, et al., "Mechanisms of Peritoneal Metastasis after Operation for NonSerosa-Invasive Gastric Carcinoma: As Ultrarapid Detection System for Intraperitoneal Free Cancer Cells and a Prophylactic Strategy for Peritoneal Metastasis," Clinical Cancer Research, Vol. 9, No. 2, 2003, pp. 678-685.

[17] M. Kuramoto, S. Shimada, S. Ikeshima, et al., "Extensive Intraoperative Peritoneal Lavage as a Standard Prophylactic Strategy for Peritoneal Recurrence in Patients with Gastric Carcinoma," Annals of Surgery, Vol. 250, No. 2, 2009, pp. 242-246. doi:10.1097/SLA.0b013e3181b0c80e

[18] Y. Kodera, Y. Yamamura, Y. Shimizu, et al., "Peritoneal Washing Cytology: Prognostic Value of Positive Findings in Patients with Gastric Carcinoma Undergoing a Potentially Curative Resection," Journal of Surgical Oncology, Vol. 72, No. 2, 1999, pp. 60-65. doi:10.1002/(SICI)1096-9098(199910)72:2<60::AID-JSO 3>3.0.CO;2-1

[19] R. Rosenberg, H. Nekarda, P. Bauer, et al., "Free Peritoneal Tumor Cells Are Independent Prognostic Factor in Curatively Resected Stage IB Gastric Carcinoma," The British Journal of Surgery, Vol. 93, No. 3, 2006, pp. 325-331. doi:10.1002/bjs.5196

[20] S. Fujimoto, M. Takahashi, T. Mutou, et al., "Successful Intraperitoneal Hyperthermic Chemoperfusion for the Prevention of Post Operative Peritoneal Recurrence in Patients with Advanced Gastric Carcinoma," Cancer, Vol. 85 , No. 3, 1999, pp. 529-534. doi:10.1002/(SICI)1097-0142(19990201)85:3<529::AIDCNCR3>3.0.CO;2-9

[21] S. Abe, H. Yoshimura, H. Tabara, et al., "Curative Resection of Gastric Cancer: Limitation of Peritoneal Lavage Cytology in Predicting the Outcome," Journal of Surgical Oncology, Vol. 59, No. 4, 1995, pp. 226-229. doi:10.1002/jso.2930590405

[22] F. Bozzetti, G. Bonfani, A. Morabito, et al., "A Multifactorial Approach for the Prognosis of Patients with Carcinoma of the Stomach after Curative Resection," Surgery, Gynecology \& Obstetrics, Vol. 162, No. 3, 1986, pp. 229-234.

[23] S. Shimada, Y. Yagi, K. Shiomori, et al., "Characterization and Proposal of the Optimal Therapeutic Strategy for Early Gastric Cancer," Surgery, Vol. 129, No. 6, 2001, pp.
714-719. doi:10.1067/msy.2001.114217

[24] J. J. Bonenkamp, I. Songun, M. Sasako, et al., "Randomised Comparison of Morbidity after D1 and D2 Dissection for Gastric Cancer in 996 Dutch Patients," Lancet, Vol. 345, No. 8952, 1995, pp. 745-748. doi:10.1016/S0140-6736(95)90637-1

[25] C. C. Wu, J. T. Chen, M. C. Chang, et al., "Optimal Surgical Strategy for Potentially Curable Serosa-Involved Gastric Carcinoma with Intraperitoneal Free Cancer Cells," Journal of American College of Surgeons, Vol. 184, No. 6, 1997, pp. 611-617.

[26] N. Hayes, J. Wayman, V. Wadehra, et al., "Peritoneal Cytology in the Surgical Evaluation of Gastric Carcinoma," British Journal of Cancer, Vol. 79, No. 3-4, 1999, pp. 520-524. doi:10.1038/sj.bjc.6690081

[27] Y. Yamamura, S. Ito, Y. Mochizuki, et al., "Distribution of Free Cancer Cells in the Abdominal Cavity Suggests Limitation of Bursectomy as an Essential Component of Radical Surgery for Gastric Carcinoma," Gastric Cancer, Vol. 10, No. 1, 2007, pp. 24-28. doi:10.1007/s10120-006-0404-5

[28] T. Mori, Y. Fujikawa, Y. Sugita, et al., "Application of Molecular Diagnosis for Detection of Peritoneal Micrometastasis and Evaluation of Preoperative Chemotherapy in Advanced Gastric Carcinoma," Annals of Surgical Oncology, Vol. 11, No. 1, 2004, pp. 14-20. doi:10.1007/BF02524340

[29] K. Maruyama, "The Most Important Prognostic Factors for Gastric Cancer Patients. A Study Using Univariate and Multivariate Analyses," Scandinavian Journal of Gastroenterology, Vol. 22, No. 133, 1987, pp. 63-68. doi: 10.3109/00365528709091021

[30] Japanese Research Society for Gastric Cancer, "Japanese Classification of Gastric Carcinoma," Kanehara, Tokyo, 1999.

[31] L. E. Harrison, M. S. Karpeh, M. F. Brennan, et al., "Extended lymphadenectomy Is Associated with a Survival Benefit for Node-Negative Gastric Cancer," Journal of Gastrointestinal Surgery, Vol. 2, No. 2, 1998, pp. 126131. doi:10.1016/S1091-255X(98)80002-4

[32] H. Zhang, C. Liu, D. Wu, et al., "Does D3 Surgery Offer a Better Survival Outcome Compared to D1 Surgery for Gastric Cancer? A Result Based on a Hospital Population of Two Decades as Taking D2 Surgery for Reference," BMC Cancer, Vol. 10, No. 20, 2010, pp. 308. doi:10.1186/1471-2407-10-308 